\title{
FABRICATION OF TRANSMISSIVE DIFFRACTIVE OPTICAL ELEMENTS FOR THE MID-INFRARED WITH A LASER WRITING INSTRUMENT
}

\author{
S. Calixto, Gil. A. Pérez-Herrera, M. Strojnik, M. Servin, Zacarias-Malacara \& Reyna A. Duarte- \\ Quiroga
}

Centro de Investigaciones en Óptica, A. C.

Apartado Postal 1-948, 37000 León, Gto., México.

scalixto@cio.mx

\begin{abstract}
A simple method to fabricate infrared $(\lambda=10.6 \mu \mathrm{m})$ diffractive optical elements that work in a transmission mode is presented. A laser-writing instrument completely under computer control has been built to demonstrate the feasibility of this method. Several diffractive elements, fabricated using the laser-writing instrument, are described.

\section{RESUMEN}

Se presenta un método sencillo para fabricar elementos ópticos difractores para infrarrojo $(\lambda=10.6 \mu \mathrm{m})$. Estos elementos trabajan en transmisión. Para la fabricación de ellos se construyó un instrumento de escritura por medio de luz láser controlado por computadora. Varios de los elementos ópticos difractores fueron evaluados.
\end{abstract}

KEYWORDS: Diffractive elements, infrared, infrared components, transmissive optics.

\section{INTRODUCTION}

Diffractive optical elements (DOE) are replacing the traditional optical elements in some applications because of their reduced thickness, weight, and superior surface finish [1]. They have been particularly important for applications in the mid-infrared region where the glass transmission is decreased [2]. In the laser beam handling, shaping, and diagnostics applications, the lenses are seldom used, because of the (thermal) absorption, heating, and damage effects [3]. Therefore, the transmissive DOEs with zero-one transmission function are highly appropriate for IR applications.

A large number of fabrication techniques have been developed in response to the real need to incorporate these diffractive elements into actual optical systems. Elements may be fabricated using any of several methods like direct interference pattern recording with UV [4], visible [5], or IR light [6], diamond turning [7], contact printing [8], laser [9, 10] and electron beam writing [11-15]. Elements fabricated using the last method may attain the highest diffraction efficiency. However, their excellent quality requires a dedicated use of an expensive fabrication technology, developed and optimized for different research applications, i.e., microelectronics.

Possibly the most significant drawback of this technique in the research environment is that it comprises multiple, time consuming steps. Additionally, the DOE quality depends on accurate positioning [16, 17] and registration of the electron beam as well as the fabrication of high quality masks [18]. Finally, the environmental problems associated with these methods of DOE fabrication are not insignificant. Certain electronic materials require careful handling because they generate health-related concerns. Also, the same piece of equipment can not be used for electronics and optical applications due to the sensitivity to 
contamination. For one-of-a-kind research applications, the high performance electron beam writing methods are not cost-effective for an individual researcher.

We describe a simple method to fabricate transmissive diffractive elements for the IR wavelength region. These elements have holes laser-written in them. They have a transmission of one at the aperture coordinates, where the IR radiation is transmitted and diffracted. Their transmission elsewhere is zero - the radiation is either reflected or weakly absorbed. After the transmission function of the optical component is calculated and binarized using a specified hole diameter and coordinates, this information is stored in a computer. A laser beam writing instrument under a PC-control is used to position the holes in a matrix fashion.

We use terminology "diffractive optical element, DOE" for the diffractive element that was designed on computer and fabricated in the laboratory, and "holographic optical elements, HOE" for those generated in the laboratory [19].

\section{CGH AND THE LASER-BEAM WRITING INSTRUMENT}

Computer generated holograms (CGH) were originally proposed as an accurate alternative fabrication method to the direct holographic recording performed in the laboratory [20]. Since this initial work, other algorithms have been described to calculate holograms using more efficient computational methods. We used one of these well-established and proven methods [21] to calculate Fourier holograms.

Figure 1 illustrates the computer simulation of the hologram generation process. Part a) shows a computergenerated object placed in one half of the input plane $\mathrm{O}\left(\mathrm{x}_{0}, \mathrm{y}_{0}\right)$. Its (two-dimensional) Fourier transform, calculated using a FFT routine, is spatially limited to a $60 \times 60$ element matrix. Finally, the transmittance function of the $\mathrm{CGH}$ is obtained by binarizing its real part. We liked to use positive values for the transmission function.

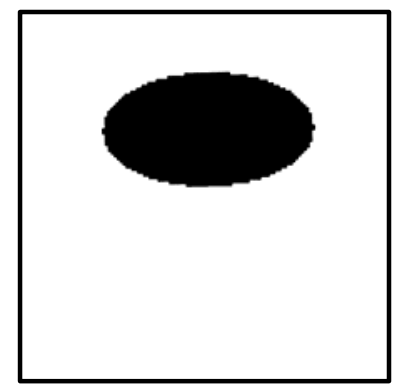

a)

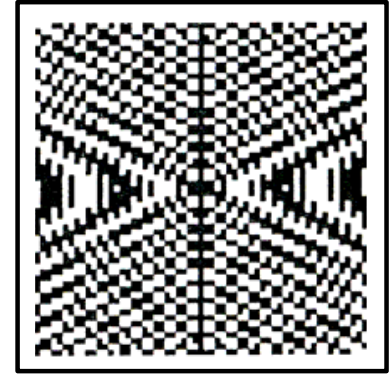

b)

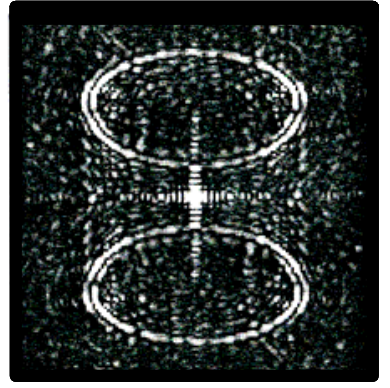

c)

Fig 1. Schematic representation of the steps followed to obtain the CGH and its reconstruction.

a) Computer-generated object, b) the CGH and c) computer reconstruction

Therefore, we re-define it over the range zero-one by adding one and dividing the sum by two:

$$
\mathrm{T}(\mathrm{x}, \mathrm{y})=\frac{1}{2}\left\{1+\operatorname{sgn}\left[\operatorname{Re}\left(\mathrm{F}\left(\mathrm{O}\left(\mathrm{x}_{0}, \mathrm{y}_{0}\right)\right)\right)\right]\right\}
$$

We use the signum function, $\operatorname{sgn}(\mathrm{x})$, as follows:

$$
\begin{aligned}
& \operatorname{sgn}(x)=1, \quad \text { if } x \geq 0 \\
& \operatorname{sgn}(x)=-1, \quad \text { if } x<0 .
\end{aligned}
$$


Here $\operatorname{Re}\{$.$\} denotes the real part of function in the curly bracket, in this case, the complex, two-$ dimensional Fourier transform, $\mathrm{F}\{$.$\} . The \mathrm{CGH}$ of the computer-generated object presented in Figure $1 \mathrm{a}$ ) is shown in Figure $1 \mathrm{~b}$ ). It consists of a square window with $60 \times 60$ binary matrix elements. The FFT calculates the Fourier transform only at discrete points, defined as the matrix coordinates. However, Figure 1b) shows the whole area associated with each pixel element as either completely black or completely white.

Any other method may be used to calculate a diffractive element. Most likely, new methods will be developed that be equally, if not more, appropriate for the computer control of the laser-beam writing instrument.

Figure 1c) shows the computer reconstruction of the binarized, transmission function, shown in Figure 1b). First, the Fourier transform of the pattern in Figure $1 \mathrm{~b}$ ) is calculated. Then, it is converted to a positive number by finding its magnitude. The large range of intensity values associated with each pixel element is compressed using the log-function, which facilitates the display of information. The intensity gradients are indicative of the sharpness of edges in the original input image. The reconstructed pattern consists of three parts. The central cross is the diffraction pattern due to the finite size of the matrix of the binarized $\mathrm{CGH}$. The single ellipse in the upper half plane of Figure 1a) is replicated in the upper and the lower half plane in the reconstruction. The ellipses contain the identical information. The double contours in the reconstructed object indicate intensity discontinuities in the original input image.

After the hologram transmission matrix has been calculated on computer, the pattern is transferred to a substrate, one pixel at a time. For this step, we built a PC-controlled laser writing device, whose diagram is shown in Figure 2. Visible (or mid-infrared) radiation is focused to a small spot on the (DOE) substrate, made of a polymer foil. The substrate position and movement is controlled with two linear, motorized, $X$, $Y$ displacement stages. Maximum substrate displacement in either axis is approximately $5 \mathrm{~cm}$. The DCcontrolled motors incorporate an integral position-encoder [22]. This motor-encoded combination has a resolution of $0.1 \mu \mathrm{m}$ and a typical backlash of less than $6 \mu \mathrm{m}$. The electronics to control the motors was custom-designed and built in our laboratory. The position of $x, y$ coordinates is controlled with a PC via a controller chip (National Semiconductors LM629). It incorporates an integral-derivative-proportional digital filter, preset for the speed and acceleration to reduce the positioning time. The actual position speed and acceleration are fed-back to the controller as an error signal. This configuration results in the optimum positioning time.

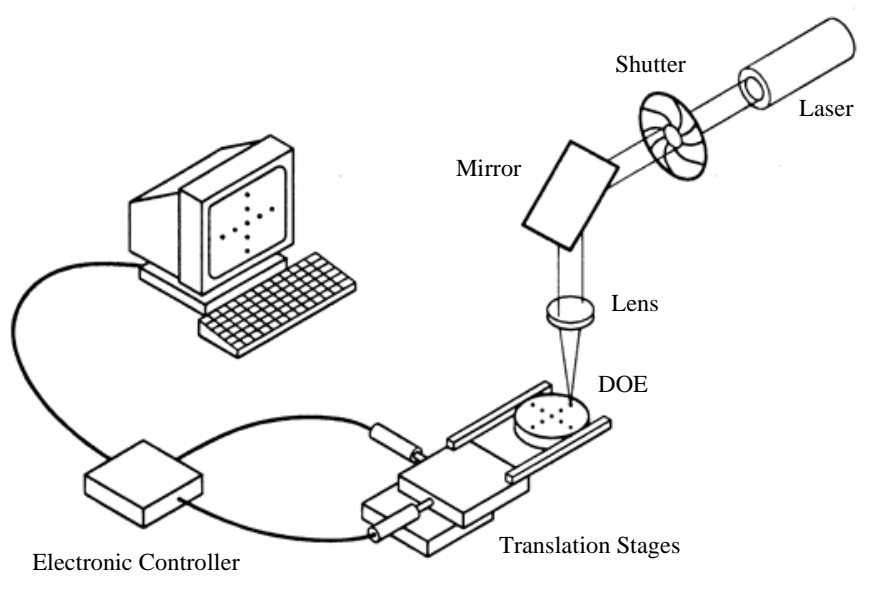

Figure 2. Diagram of the laser-beam writing instrument 
The calculated, binary, transmittance function $T(x, y)$ is converted in software to the hole coordinates using specified hole diameters. Currently, the software accepts input data for the DOE with $60 \times 60$ pixels. Pixel separation may be selected by the operator, according to the application. So far, we have used the device with two representative pixel distances of $20 \mu \mathrm{m}$ and $150 \mu \mathrm{m}$. The pixel separation determines the DOE fabrication time. The recording time for $20 \mu \mathrm{m}$ pixel separation is approximately 6 hours in a step-by-step method. It increases to nearly 18 hours when a $150 \mu \mathrm{m}$ pixel separation is employed. It is expected that an appreciable reduction in the DOE recording time will be realized when a continuously-scanning mode is implemented. This work has started recently.

The knowledge of positioning the hole centers is maintained within the interferometric accuracy. Two Michelson interferometers, one for each axis, are used to calibrate the accuracy and precision of the positioning device. This setup is shown in Figure 3 for a single axis only. A mirror in one arm of the Michelson interferometer is attached to the moving stage while the other mirror remains stationary. A He$\mathrm{Ne}$ laser is used as a coherent source in these two-beam interferometers. When the stage moves incrementally, fringes are counted to obtain the corresponding measured substrate displacement $\Delta \mathrm{s}$.

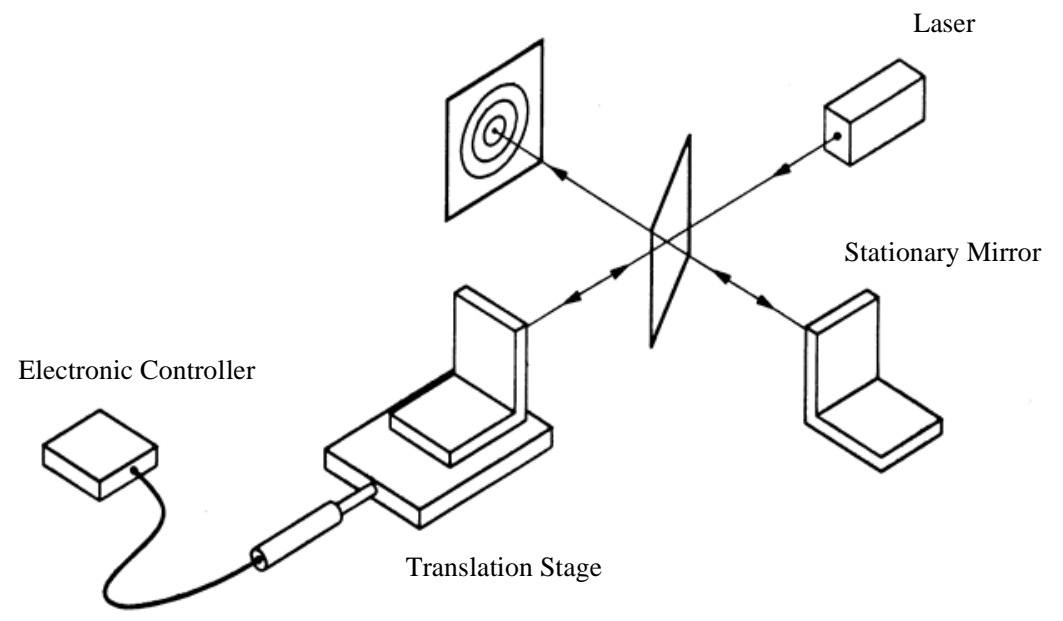

Figure 3. Opto-mechanical setup used to measure stage displacement

The calculated position has been calibrated against the measured substrate movement in a calibration experiment. The PC-controlled linear displacement has been recorded for a series of points at $50 \mu \mathrm{m}$ position increments. (The increment number is denoted by $n$ ). Concurrently, the interference fringes have been counted. Figure 4 shows the measured incremental displacement $\Delta s$ vs. the increment number, $\mathrm{n}$. This graph shows a cyclic error of $\pm 4 \mu \mathrm{m}$ between the interferometer-measured displacement and the PC-controlled position. The cumulative error is actually much smaller than that indicated in Figure 4. This is illustrated in Figure 5, showing the total distances traveled by the moving mirror as a function of $n$, the distance increment number $n$. The curve linearity demonstrates the accuracy of positioning the hole centers using the PC-controlled laser-writing device. 


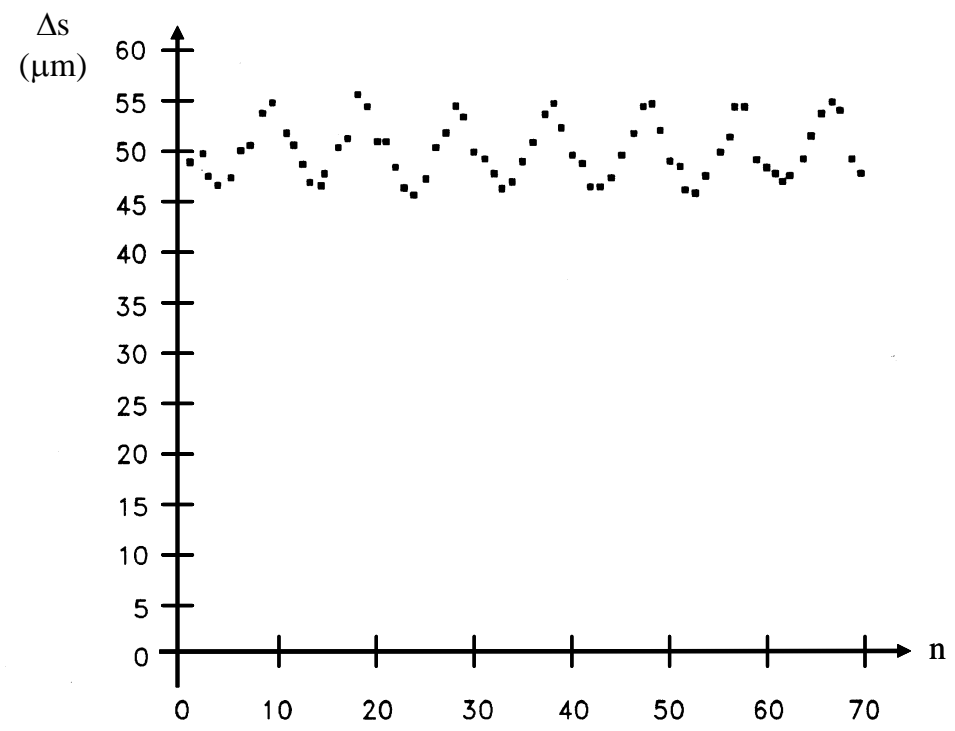

Figure 4. Effective displacement $\Delta s$ as measured by the Michelson interferometer for every $50 \mu m$ displacement programmed on the computer. $n$ is the sequential $50 \mu \mathrm{m}$ displacement

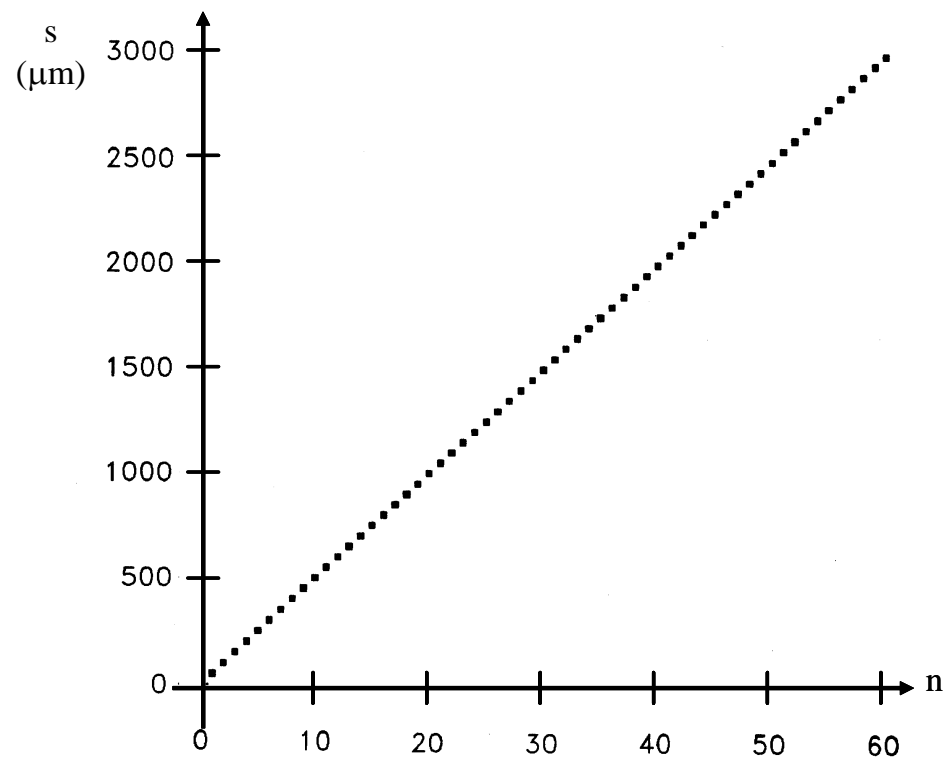

Figure 5. Distance $s$ traveled by the mirror on the stage as a function of the desired distance programmed in the computer. Each integer $n$ corresponds to $50 \mu \mathrm{m}$ displacement 


\section{COMPLEX STRUCTURES - RECORDING AND RECONSTRUCTION WITH VISIBLE LIGHT}

The computer-generated object, shown in Figure 6a), was used as the input for the calculation of the DOE transmission function. It consists of three letters of the alphabet resembling three types of lenses having ellipses and ellipse segments as boundaries. The Fourier hologram of this input object was generated on a $60 \times 60$ pixel matrix and binarized. The corresponding computer-reconstructed intensity pattern is shown in Figure 6b). It includes the same three features discussed in reference to Figure 1c): the central intensity cross corresponds to the window used to display the binarized $\mathrm{CGH}$, and the two identical reconstructions of the input object are symmetrical across the coordinate origin assumed at the center of the square diffraction pattern. The computer reconstruction was also performed to create a reference image in order to assess the quality of the DOE and its optical reconstruction.

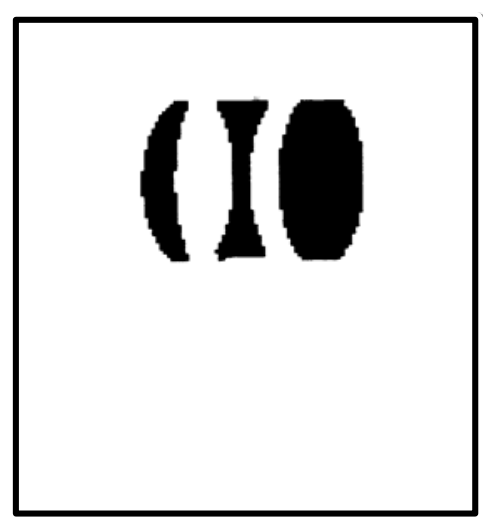

(a)

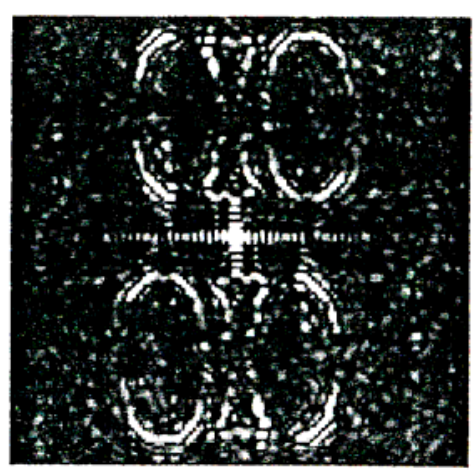

(b)

Figure 6. Object made on the computer and used to calculate the Fourier hologram in a $60 \times 60$ pixels matrix.

b) Reconstruction made by the computer

After the matrix had been calculated, its DOE was recorded with a He-Ne laser on a holographic film plate. A microphotograph of the recorded pattern is shown in Figure 7a), with a $10 \mu \mathrm{m}$ pixel size. Optical reconstruction, also made with a visible light, is shown in Figure $7 \mathrm{~b}$ ). There is some resemblance between the optically-reconstructed pattern of Figure $7 \mathrm{~b}$ ) and the computer-reconstructed pattern of Figure 6b).

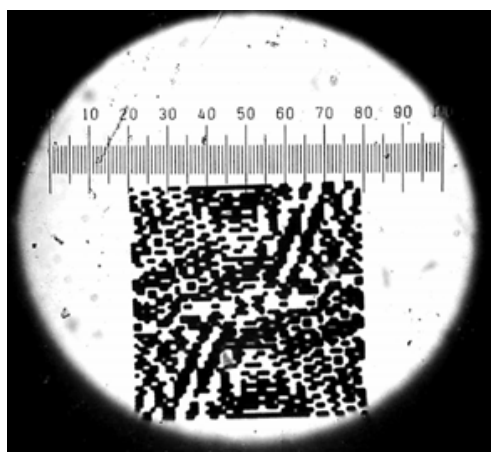

a)

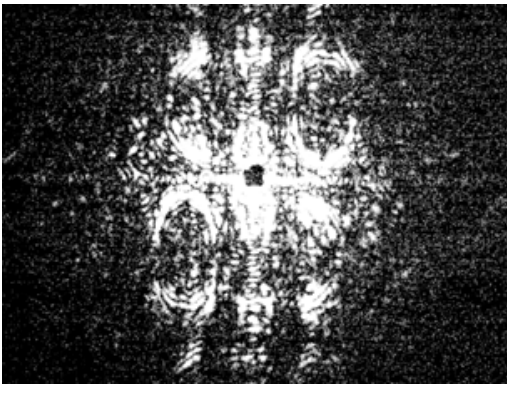

b)

Figure 7. a) Microphotograph of the CGH written with He-Ne laser light in a holographic plate by means of the laser beam writing device. Distance between pixels is about $20 \mu \mathrm{m}$. The distance between two adjacent lines in the scale is $10 \mu \mathrm{m}$.

b) Reconstruction of the hologram done with visible light 
There are some reasons for the differences between Figures $6 b$ ) and $7 b$ ): Figure $6 b$ ) has been displayed originally on a hardware (monitor/printer) with the display threshold and gamma under operator control. The optically-reconstructed intensity pattern of Figure 7b), on the other hand, has been generated using sharp intensity spots of finite dimensions. Additionally, the film used to record the optically-reconstructed image has been chosen for its high resolution and sensitivity, recording the most minute intensity details; these, unfortunately, include also the noise.

\section{COMPLEX STRUCTURES - RECORDING WITH MID-INFRARED LIGHT}

In the preceding sections, we have described some characteristics of the diffractive elements using visible light for the recording and the reconstruction steps. The transfer of the technology, developed and demonstrated in the visible wavelength region, to the infrared requires three additional ingredients: first, the use of a mid-IR laser to record the pixels as holes; second, the existence of a suitable target material on which good quality holes could be laser-written; and, third, the availability of a detector material sensitive in the IR region to display the image produced by the DOE.

A setup identical to the one used for the visible light was used (Figure 2), but this time a germanium lens was used to focus a $\mathrm{CO}_{2}$ laser beam $(10.6 \mu \mathrm{m})$ on the target. The preprogrammed hole pattern was burnt into a polymer film [6] to create the DOE. First, we obtained the response of the polymer films to the $\mathrm{CO}_{2}$ irradiation. The film thickness ranged from $40 \mu \mathrm{m}$ to $100 \mu \mathrm{m}$. For each film thickness, several exposures were made to determine experimentally the optimum conditions to create holes with constant diameter and uniform separation. The hole diameters vary from $10 \mu \mathrm{m}$ to $200 \mu \mathrm{m}$. However, for several materials/exposure combinations, irregularly-shaped holes were generated. In some instances, the hole boundaries were not well defined and clean - debris was found on the outside of the holes. This results in a poor interhole separation, producing a large amount of scattered light. Other deformation resulting from the IR laser irradiation includes the formation of a doughnut-shaped bump outside the holes.

The micropositioning device can displace the substrate by linear increments as small as $10 \mu \mathrm{m}$. However, due to the hole dimensions and the film distortions, a high quality hole pattern was recorded with a $150 \mu \mathrm{m}$ pixel separation and a hole diameter of about $20 \mu \mathrm{m}$. One such example pattern is shown in Figure 8a). To minimize the deformation of the IR-generated DOE during the reconstruction process, polymer films were aluminized after the hole pattern had been written in them. Aluminum film was used due to its high (IR) reflection and high thermal conductivity to increase heat transfer from the irradiated area.

When the DOE was illuminated with a He-Ne laser, the image shown in Fig. 8b) was obtained. 


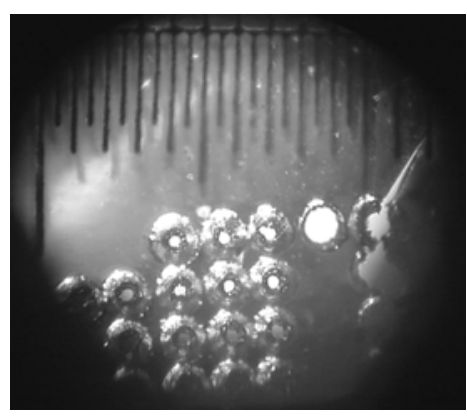

a)

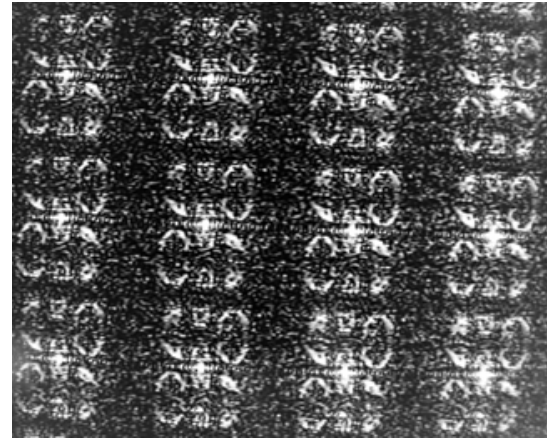

b)

Figure 8. a) DOE written with IR light in a polymeric thin film. Recording was done by means of the laser beam writing device. Every pixel is a hole. Distance between holes is about $150 \mu \mathrm{m}$. Distance between two adjacent lines in the scale shown is $50 \mu \mathrm{m}$. b) Reconstruction of DOE in a) given by He-Ne laser light (transmission mode)

The advantages of laser-writing in IR are illustrated clearly when comparing the images of Figures $7 \mathrm{~b}$ ) and $8 b$ ), both reconstructed with the visible light. It is seen that an improvement in the image quality is achieved and, therefore, that the higher DOE quality is attained with IR lasers and materials.

An additional significant observation to be made from Figure 8b) is the absence of scattered light, indicating that the hole generation in IR results in good quality DOEs. Due to the low amount of scattering, a large number of orders is seen in Figure $8 b$ ). Here, twelve orders are actually recorded due to the available film size, although more orders were observed in the laboratory. Each order includes a cross and two identical reconstructed images, resembling the computer-reconstructed pattern of Figure $6 b)$. The large number of orders is a consequence of the finite hole area in the DOE matrix. With this reconstruction process, multiple images were obtained experimentally [23, 24].

Two other interesting applications of this method deserve to be mentioned. First, an attempt has been made to increase the far-field image brightness by increasing the hole diameter from $20 \mu \mathrm{m}$ to about 90 $\mu \mathrm{m}$. A part of one such laser beam-written pattern is shown in Figure 9a.

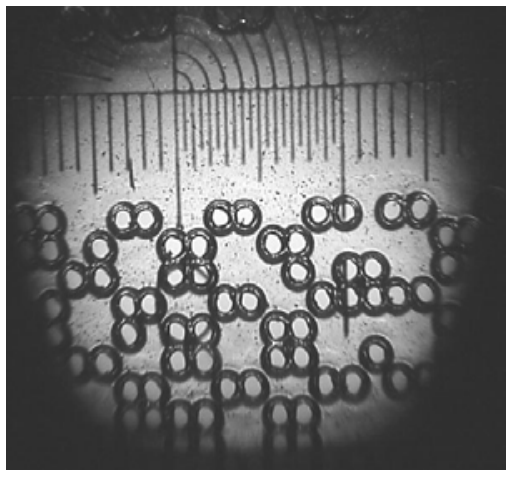

a)

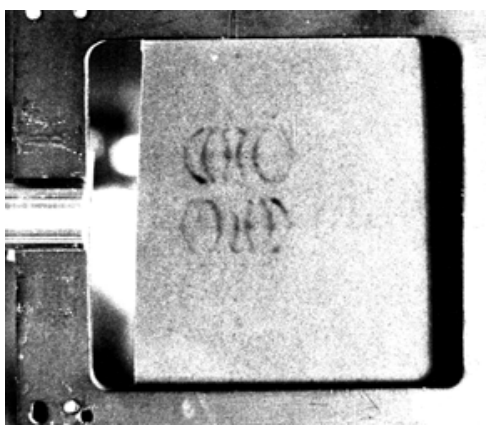

b)

Figure 9. a) Part of an IR laser beam written DOE. b) IR optical reconstruction of DOE in a). Thermal image was detected by liquid crystals. Central part of the image was blocked by a mirror. Setup to make the reconstruction is shown in Fig. 10 
The setup shown in Figure 10 was employed to perform the optical reconstruction of this DOE. A $\mathrm{CO}_{2}$ laser beam illuminates the DOE. A Ge lens, immediately behind the DOE, performs the Fourier transform. A layer of a liquid-crystal material was used for the visualization of the thermal image diffracted by the DOE. A small aluminum mirror was placed on the optical axis, at the image plane, to reflect and to filter spatially the low spatial frequencies, where the high intensity cross introduces the noise pattern due to finite matrix size.

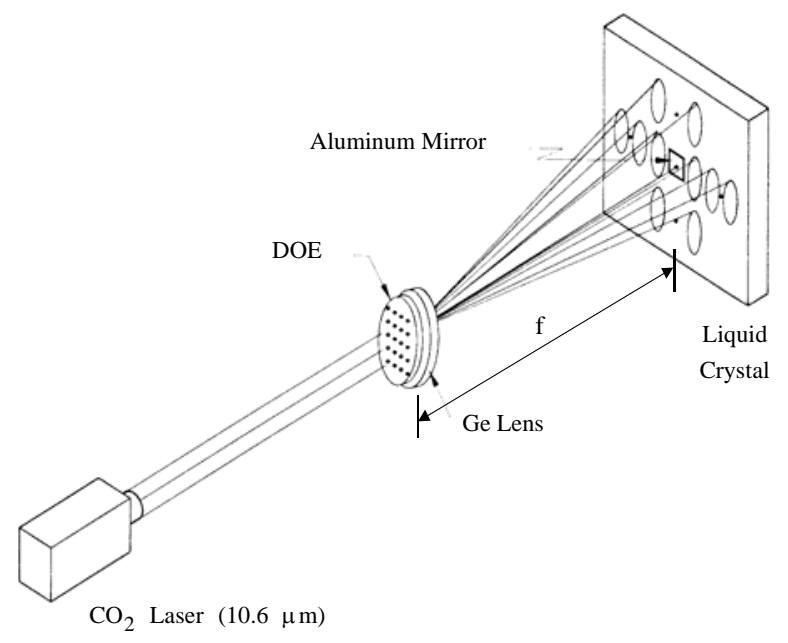

Figure 10. Setup used to make the IR image reconstruction of the DOE

Figure 9b) shows the corresponding detected infrared intensity pattern. This pattern recreates the computer simulation displayed in Figure 6b), except that the intensity cross on the optical axis has been eliminated. Figure 9b) shows just one diffracted order because the intensity of higher orders is too low to generate a response in the liquid crystal layer. Comparing the experimental pattern of Figure 9b) with the computer prediction of Figure 6b), we observe that a well defined pattern is experimentally reconstructed, with only a minimum amount of scattered light. Also, if we compare the IR-image of Figure $9 \mathrm{~b})$ with the visible one of Figure $7 \mathrm{~b}$ ) obtained from the DOE recorded in a standard holographic filmplate, the IR image shows an improvement. These two diffracted images demonstrate clearly the advantages of working with infrared materials in the transmission mode. A good reconstruction of the input object is obtained - it is sharp and clearly defined. The single reconstructed order shows only the input object and its duplicate.

The second example that is of interest used the ellipse of Figure 1a) as the input object. A part of the DOE written by the IR laser beam is shown in Figure 11a). This part corresponds to the lower central part of the pattern in Figure $1 b$ ). Its optical reconstruction using the IR light is shown in Figure 11b). The IR image consists of 4 ellipses. The two in the central part belong to the first order and each one of the two remaining ones are parts of the $+/$ - second orders. Again a small mirror was placed on the optical axis to deflect and eliminate the high intensity diffraction peaks that might otherwise damage the liquid crystal material. The image in IR includes a large number of orders, similarly to the image in Figure 8b), except that now the basic pattern in each order is that shown in Figure 1c). It includes the central intensity peak representing the high intensity cross and two ellipses, located symmetrically with respect to the coordinate origin assumed at the cross. Part of the thermal image shown in Fig. 11b) was studied with an IR CCD camera. The image given by this camera can be seen in Fig. 11c). 


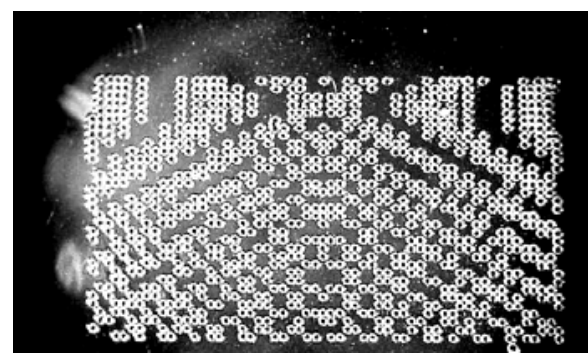

a)

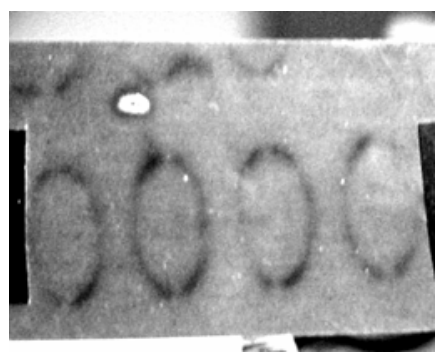

b)

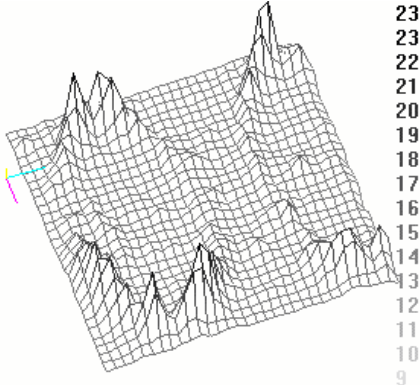

c)

Figure 11. a) Part of an IR laser beam written hologram, each hole represents a pixel of the CGH. b) IR optical reconstruction of hologram in a). Thermal image was detected by liquid crystals. 4 ellipses can be seen. Central part of the image was blocked by a mirror to avoid damage of the liquid crystals. c) Part of the IR image seen with an IR CCD camera

\section{DISCUSSION}

The diffracted elements presented here produced a rather low (spatial) resolution image because their transmission function was written with only $60 \times 60$ matrix elements. The resolution of the diffracted image, however, can be improved by using a larger number of resolution elements. This improvement will be realized when a DOE is constructed with an increasing number of matrix elements. This has been simulated initially with a computer for $120 \times 120$ matrix elements.

The computer reconstruction of the DOE of the object in Figure 6a), with a matrix of $120 \times 120$ pixels, is shown in Figure 12b). (Figure 12a includes a copy of the simulated image obtained with only $60 \times 60$ pixels to facilitate the comparison). The computer-simulated image with an increased number of pixels shows better fidelity to the input image of Figure 6a) and a low level of noise. Upon visual comparison of two parts in Figure 12, we see that the four-fold increase of the number of pixels results in an improvement in the DOE image quality. This improvement can be implemented, for example, by expanding the range of the micropositioning fixture(s).

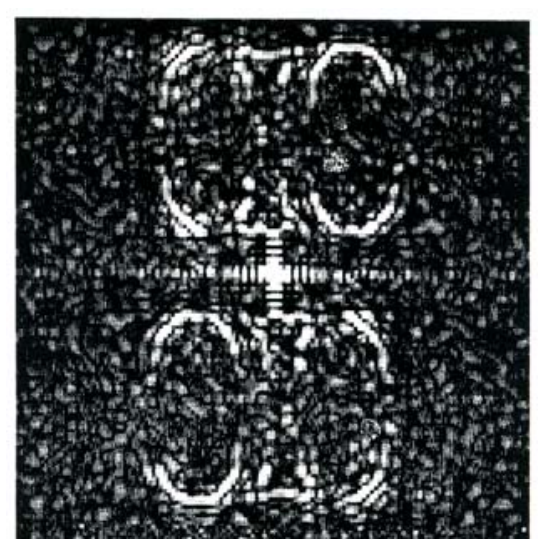

a)

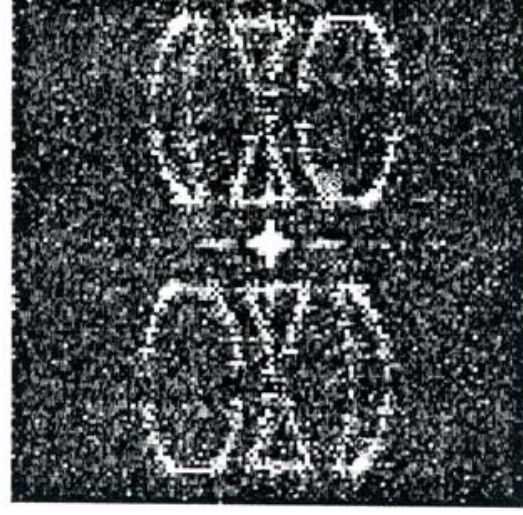

b)

Figure 12. Computer reconstructions of CGHs. In a) the matrix where the object was embedded was 60 $\times 60$ pixels. In $b$ ) the matrix was $120 \times 120$ pixels 
The size limitations of the electron beam writing devices do not apply to the laser-beam writing DOEs. The DOE size limitations may be overcome easily when employing a laser-beam writing setup, by using a larger DOE substrate that is displaced with a micropositioning device with a larger range.

Previous efforts to fabricate the transmissive diffractive IR elements [25, 26] (zone plates) relied on the surface relief methods. The function of these elements, fabricated in germanium (Ge) and gallium arsenide (GaAs), was to focus the incoming radiation to a small spot. The GaAs plate incorporated flat, multilevel relieves to attain high diffraction efficiencies. The materials used for fabricating the IR transmissive diffractive elements have high index of refraction. Their surfaces had to be covered with an antireflection coating to decrease Fresnel loses at least for one angle of incidence.

Diffracted elements fabricated with the micropositioning setup described here do not require antireflection coatings to increase the transmission of radiation at a specific wavelength. After the holes have been laser-drilled into the recording materials, generating a zero-one transmission function, a thin layer of aluminum may be evaporated on the polymer surface. This is done solely to maximize the amount of reflected light, to enhance the heat conduction away from the irradiated area, and to increase the structural strength of the DOE.

The actual amount of energy transmitted to the image plane is not considered to be of particular significance for the DOEs with zero-one transmission function. The laser-beam-written DOEs, working in a transmission mode, are characterized as having a binarized transmission. A given fraction of the radiation, given by the hole-fill factor, is reflected back to the source and does not contribute to the IR image formation. However, if a specific amount of energy is required for the image formation, then either a higher power $\mathrm{CO}_{2}$ laser may be used to write on a stronger material; that withstands higher power densities, at the reconstruction step or a lower fill factor may be used in the DOE. The use of higher energy density at the reconstruction time is expected to result in a better quality image reconstruction.

\section{CONCLUSIONS}

Up to now, transmissive diffractive IR elements have been used primarily to focus energy into a small spot. In addition to their function as imaging optical elements, we are using them also for object reconstruction.

The laser-beam writing method described here is quite similar to the well-established electron-beam writing methods. However, the laser-beam written DOEs working in the transmission mode have a number of advantages over the electron beam written devices. These include the ability to control the amount of energy transmitted to the image plane, a small number of fabrication steps, and simplicity and versatility of the experimental setup. The amount of energy transmitted to the image plane is selected with the choice of the hole-fill factor.

We summarized next the fabrication advantages of a laser-beam writing system as compared to that incorporating the electron beam devices. In both cases, the transmission pattern is calculated first, and then it is transferred onto the substrate. When the laser beam writing method is used, this step results in the finished DOE. When the electron beam is used, the substrate needs to be further processed: it is further exposed to the dry etching development to generate the diffractive element [13-16].

Additionally, the multi-step electron beam methods use several fabrication facilities developed for microelectronics applications and available for use by a large number of technicians. The probability of committing an error increases with the number of processing steps and the number of humans involved in processing. The method described here contains only a few steps, appreciably decreasing the probability of occurrence of an error. 
Possibly the greatest disadvantage of an electron-beam writing method is the maximum size of a diffractive element that can be constructed. It is equipment-restricted $[11,12]$ to only $2 \times 2 \mathrm{~cm}$. With the laser-beam writing setup, light is simply focused by a lens into a small spot. It is the substrate that is moved every time the pixel is recorded when the micropositioning device controls the differential displacements.

The technique to fabricate transmissive diffractive elements using a laser micropositioning and writing instrument is a simple and cost effective alternative to the previously published techniques

\section{REFERENCES}

[1] Missig M.D. and Morris G.M., Diffractive Optics Applied to Eyepiece Design, Applied Optics, Vol. 34, No. 14, May, 1995, pp. 2452-2461.

[2] Hudson R.D., Jr., Optics, in Infrared System Engineering, John Wiley \& Sons, 1969, pp. 216-217.

[3] Marquet L.C., Transmission Diffraction Grating Attenuator for Analysis of High Power Laser Beam Quality, Applied Optics, Vol. 10, No. 4, April, 1971, pp. 960-961.

[4] Ilcisin K.J. and Fedosejevs R., Direct Production of Gratings on Plastic Substrates Using 248-nm KrF Laser Radiation, Applied Optics, Vol. 26, No. 2, January, 1987, pp. 396-400.

[5] Ming H., Wu Y., Xie J. and Nakajima T., Fabrication of Holographic Microlenses Using a Deep UV Lithographed Zone Plate, Applied Optics, Vol. 29, No. 34, December, 1990, pp. 5111-5114.

[6] Calixto S., Polymers as Mid-Infrared Holographic Recording Media, Polymers in Information Storage Technology, K.L. Mital, ed., Plenum Press, 1989, pp. 217-225, New York, U.S.A., February.

[7] Clark P.P. and Londono C., Production of Kinoforms by Single Point Diamond Machining, Optics News, Vol. 15, No. 12, December, 1989, pp. 39-40.

[8] Boiko Y., Solovjev V.S., Calixto S. and Lougnot D.J., Dry Photopolymer Films for ComputerGenerated Infrared Radiation Focusing Elements, Applied Optics, Vol. 33, No. 5, February, 1994, pp. 787-791.

[9] Gale M.T., Rossi M., Schutz H., Ebets P., Herzig H.P. and Prongue D., Continuous- Relief Diffractive Optical Elements for Two-Dimensional Array Generation, Applied Optics, Vol. 32, No. 14, May, 1993, pp. 2526-2533.

[10] Krackhardt U.W., Schwider J., Schrader M. and Streibl N., Synthetic Holograms Written by a Laser Pattern Generator, Optical Engineering, Vol. 32, No. 4, April, 1993, pp. 781-785.

[11] Fujita T., Nishihara H. and Koyama J., Fabrication Of Micro Lenses Using Electron-Beam Lithography, Optics Letters, Vol. 6, No. 12, December, 1981, pp. 613-615.

[12] Fujita T., Nishihara H. and Koyama J., Blazed Gratings and Fresnel Lenses Fabricated by Electron-Beam Lithography, Optics Letters, Vol. 7, No. 12, December, 1982, pp. 578-590. 
[14] Newman D.M., Hawley R.W., Goeckel D.L., Crawford R.D., Abraham S. and Gallagher N.C., Efficient Storage, Computation, and Exposure of Computer Generated Holograms by Electron Beam Lithography, Applied Optics, Vol. 32, No. 14, May, 1993, pp. 2555-2565.

[15] Daschner W., Larsson M. and Lee S.H., Fabrication of Monolithic Diffractive Optical Elements by the Use of e-Beam Direct Write on an Analog Resist and a Single Chemically Assisted Ion-Beam-Etching Step, Applied Optics, Vol. 34, No. 14, May, 1995, pp. 2534-2539.

[16] Cox J.A., Fritz B. and Werner T., Process Error Limitations on Binary Optics Performance, Computer and Optically Generated Holographic Optics, 1991, pp. 80-88, Bellinsham, Washington, U.S.A.

[17] Miller J. M., Taghizadeh M.R., Turunen J. and Ross N., Multilevel-Grating Array Generators: Fabrication Error Analysis And Experiments, Applied Optics, Vol. 32, No. 14, May, 1993, pp. 2519-2525.

[18] Duparre M., Golub M.A., Ludge B., Pavelyev V.S., Soifer V.A., Uspleniev G.V. and Volotovskii S.G., Investigation of Computer-Generated Diffractive Beam Shapers for Flattening of SingleModal $\mathrm{CO}_{2}$ Laser Beams, Applied Optics, Vol. 34, No. 14, May, 1995, pp. 2489-2497.

[19] Feldman M.R., Diffracted Optics Moves into the Commercial Arena, Laser Focus, Vol. 30, No. 6, June, 1994, pp. 145-151.

[20] Brown B.R. and Lohmann A.W., Complex Spatial Filtering with Binary Masks, Applied Optics, Vol. 5, No. 6, June, 1966, pp. 967-969.

[21] Bryngdahl O. and Wyrowski F., Digital Holography-Computer-Generated Holograms, Progress in Optics XXVII, 1990, pp. 220-252, Amsterdam, North Holland.

[22] Oriel Corporation, Stratford, CT, USA.

[23] Metha P.C., Bahn C. and Hraydanath R., Multiple Imaging by Lensless Fourier Transform Holography, Journal of Optics, Vol. 10, No. 4, February, 1979, pp. 133-137.

[24] Calixto S., Dry Polymer for Holographic Recording, Applied Optics, Vol. 26, No. 18, September, 1987, pp. 3904-3910.

[25] Hasman E., Davidson N. and Friesem A.A., Efficient Multilevel Phase Holograms for $\mathrm{CO}_{2}$ Lasers, Optics Letters, Vol. 16, No. 6, March, 1991, pp. 423-425.

[26] Hutley M.C., Stevens R.F. and Wilson S.J., Manufacture of Blazed Zone Plates in Germanium for Use in the $10 \mu \mathrm{m}$ Spectral Region, Optical Engineering, Vol. 30, No. 7, July, 1991, pp. 1005-1010

\section{ACKNOWLEDGEMENTS}

Authors thank Daniel Malacara for helpful discussions. Additionally, Norma Munoz's assistance with software, controller design, and fabrication is greatly appreciated. We also thank Octavio Pompa for deposition of aluminum films. 
Authors Biography

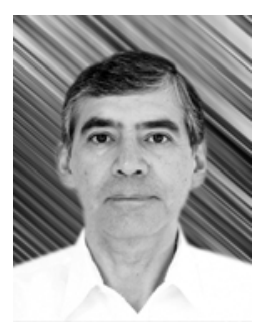

Sergio Calixto

Sergio Calixto received his B.S. degree in physics from the National University of Mexico, his M.S. degree in optics from the National Institute of Astrophysics, Optics and Electronics, Mexico, a Master in Philosophy degree in optics from the Imperial College of Science and Technology, England, and his Ph.D. from Laval University, Canada. His research interests include holographic recording materials and micro-optical fabrication materials and methods. He is a member of SPIE and OSA.

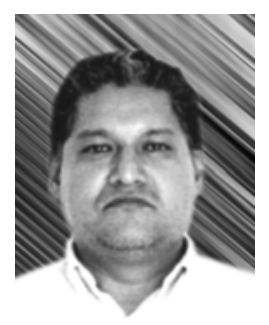

\section{Gil A. Perez-Herrera}

Gil A. Perez Herrera received his bachelor's degree in electronics from the Universidad de Guanajuato, and his M.Sc. in optics from the Centro de Investigaciones en Óptica, A.C. Currently, he works at the Centro de Investigaciones en Óptica (Aguascalientes).

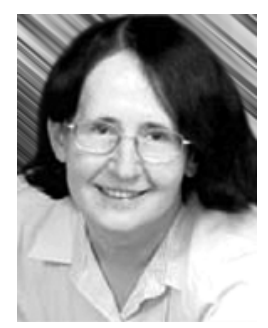

Marija Strojnik-Pogacar

Dr. Marija Strojnik developed an optical navigation technique fully autonomic based on the recognition of stellar fields by using an intelligent camera. She is also interested in discovering planets outside our solar system. She was topic editor of Applied Optics and invited editor of Optical Engineering and currently she is fellow of the OSA and SPIE. 


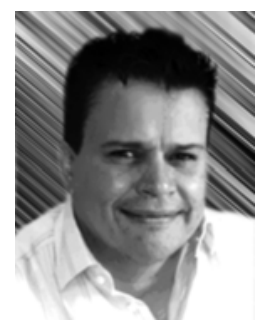

\section{Manuel Servin-Guirado}

Manuel Servin Guirado, obtained his doctoral degree from the Centro de Investigaciones en Óptica A,C. Leon, Guanajuato, Mexico in May 1994. He is a specialist in fringe pattern processing and interferometry and has published over 70 scientific papers in this field. Over the last few years, he has collaborated with the Editorial Board in the SPIE annual meeting conference on Interferometry.

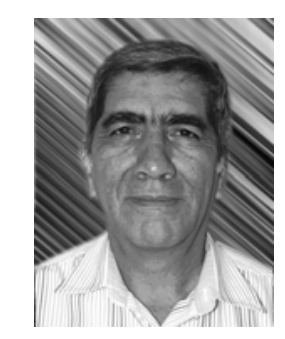

\section{Zacarías Malacara}

Zacarías Malacara holds a bachelor's degree from the National University of Mexico and a doctoral degree from the Universidad de Guanajuato in Mexico. He currently works in the Optical Engineering Department at the Centro de Investigaciones en Óptica in Leon, Mexico, doing research on optical instrumentation. His interests also include color measurements and optical designing.

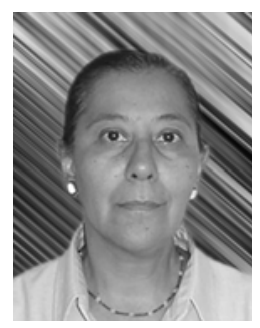

Reyna A. Duarte-Quiroga

Reyna A. Duarte-Quiroga received her B.S. degree in physics from the Universidad Nacional Autónoma de México and her M.Sc. and Ph.D. from Centro de Investigaciones en Óptica, A.C., Leon, Guanajuato, Mexico. Her research interests include photosensitive recording materials and holography. She has been in charge of the Posgraduate Students Labs in the Centro de Investigaciones en Óptica, A.C. since January 2002. 